\title{
THEORETICAL DESCRIPTION OF SHELL FILLING IN CYLINDRICAL QUANTUM DOTS
}

\author{
B. Szafran, S. Bednarek and J. Adamowski \\ Faculty of Physics and Nuclear Techniques, Technical University (AGH) \\ Al. Mickiewicza 30, 30-059 Kraków, Poland
}

\begin{abstract}
A theoretical quantitative description is given for non-periodic oscillations observed in a current vs. gate voltage characteristics of cylindrical quantum dots. Energy eigenvalues and chemical potentials for a system of $N$ confined electrons $(N=1, \ldots, 13)$ are calculated by the unrestricted Hartree-Fock method with a confinement potential proportional to the gate voltage. The positions of the observed peaks of the current are reproduced with a very good accuracy. The non-periodicity of the characteristic is explained as a result of the shell filling, which is a signature of the quantum Coulomb blockade.
\end{abstract}

PACS numbers: 73.20.Dx

Recently, the non-periodic pattern of peaks has been observed [1] in the current-gate voltage characteristics of InGaAs/AlGaAs cylindrical quantum dots (QDs). This property cannot be explained by the classical Coulomb blockade model [2] and is tentatively attributed [1] to a filling by electrons of the shells in an artificial atom, which is formed in the QD region. According to the classical Coulomb blockade model, the energy, which is necessary to add one electron to the $\mathrm{QD}$, is approximately equal to the charging energy of a capacitor, which corresponds to the QD. This model predicts the equal spacing between the peaks of the current observed as a function of the gate voltage, i.e., the periodic pattern of peaks. Such a behavior was observed in the QDs, which contained a large number (several tens) of electrons [2]. The new feature observed by Tarucha et al. [1] resulted from the possibility of decreasing the number of electrons in the $\mathrm{QD}$ to 0 by increasing the gate voltage. For a small number of electrons, the current-gate voltage characteristic clearly shows the quantum properties of the electron system. Ezaki et al. [3] calculated the electronic structure in circular quantum dots, which exhibit some similarity to the fabricated nanostructures [1]. An attempt of theoretical description of the experimental results [1] has been undertaken by Nagaraja et al. [4]. The authors approximated the real cylindrical nanostructure [1] by a rectangular box, which yielded the results that only qualitatively corresponded to the experimental data [1]. In the present work, we provide a quantitative quantum-mechanical description of the observed [1] shell-filling effects. For this purpose, we study the many-electron system, which is subjected to 
the three-dimensional gate-voltage-dependent confinement potential of cylindrical symmetry.

The cylindrical QD [1] is determined by the ring-shaped side-gate electrode and two vertically placed $\mathrm{Al}_{0.22} \mathrm{Ga}_{0.78} \mathrm{As}$ barriers. The inner $\mathrm{QD}$ region is made of $\mathrm{In}_{0.05} \mathrm{Ga}_{0.95} \mathrm{As}$, its radius is $250 \mathrm{~nm}$, the barriers are spaced by $12 \mathrm{~nm}$. The source and drain electrodes are mounted at the upper and lower sides of the cylinder. The entire nanostructure is grown on the GaAs substrate. In order to describe the excess electrons in this QD, we apply the effective-mass approximation and assume that the confinement potential acting on electrons possesses the cylindrical symmetry and can be separated into the vertical $\left[V_{\|}(z)\right]$ and lateral $\left[V_{\perp}(\rho)\right] \mathrm{com}$ ponents. [We denote the cylindrical coordinates by $(\rho, z, \phi)$.] The potential $V_{\|}(z)$ is taken on as the rectangular potential well of finite depth, i.e., the corresponding potential energy $U_{\|}(z)$ of electrons takes on the values: $U_{\|}(z)=-180 \mathrm{meV}$ inside the QD, i.e., for $|z|<6 \mathrm{~nm}$, and $U_{\|}=0$ otherwise. The lateral confinement results from the negative voltage applied to the gate electrode, which repulses the electrons towards the QD axis and simultaneously raises the potential energy of the electrons in the QD with respect to the Fermi level of the source-drain system. We assume that the electron potential energy resulting from the lateral confinement is proportional to the gate voltage $V_{\text {gate }}$ and has the form

$$
U_{\perp}(\rho)=e V_{\text {gate }}\left(\alpha+\beta \rho^{2}+\gamma \rho^{4}\right),
$$

where $e$ is the charge of electron, $\alpha, \beta$, and $\gamma$ are treated as adjustable parameters. The form of the confinement potential used in the present paper is suggested by the approximate analytical solutions of the Poisson equation for the considered nanostructure. The conduction-band minimum of the AlGaAs barrier in the absence of the gate voltage is set equal to zero and taken as the reference energy.

We consider the system of $N$ electrons, which are subjected to the confinement potential and interact between themselves via the screened Coulomb interactions. The changes of the dielectric constant $\varepsilon$ and the electron effective band mass $m_{e}$ at the dot/barrier interface have been neglected. We take on the values of these material parameters, which correspond to the $\operatorname{In}_{0.05} \mathrm{Ga}_{0.95} \mathrm{As}$ alloy, i.e., $\varepsilon=10.96$ and $m_{\mathrm{e}} / m_{\mathrm{e} 0}=0.065$.

We solved the eigenvalue problem for $N$ interacting electrons $(N=1, \ldots, 13)$ confined in the QD with the help of the self-consistent unrestricted Hartree-Fock method. More details about the application of the Hartree-Fock method to quantum dots can be found, e.g., in Ref. [5]. In the present work, the one-electron orbitals were taken on as the linear combinations

$$
\exp \left(-b z^{2}\right) \sum_{m n p} c_{m n p} x^{m} y^{n} \exp \left[-a\left(x^{2}+y^{2}\right) / p\right],
$$

where $a, b$, and $c_{m n p}$ are the variational parameters, $x^{2}+y^{2}=\rho^{2}$, and the other parameters take on the following values: $m, n=0, \ldots, 3$, and $p=1, \ldots, 4$. The $z$-dependent part of the wave function corresponds to the ground state only, since the energy-level separations resulting from the size quantization in the $z$-direction are large due to the small thickness of the InGaAs layer. Therefore, for the consid- 
ered numbers of electrons $(N \leq 13)$, only the ground state for the motion in the $z$-direction is occupied. For $N=13$, the convergence of the variational procedure was achieved already for 28 elements used in expansion (2).

The ground-state energies $E_{N}$ for $N$ electrons in the QD calculated as functions of the gate voltage $V_{\text {gate }}$ allow us to obtain the chemical potentials according to the formula: $\mu_{N}=E_{N}-E_{N-1}$. Using the calculated chemical potentials, we find the position of peaks in the current-gate voltage characteristics (Fig. 1). For

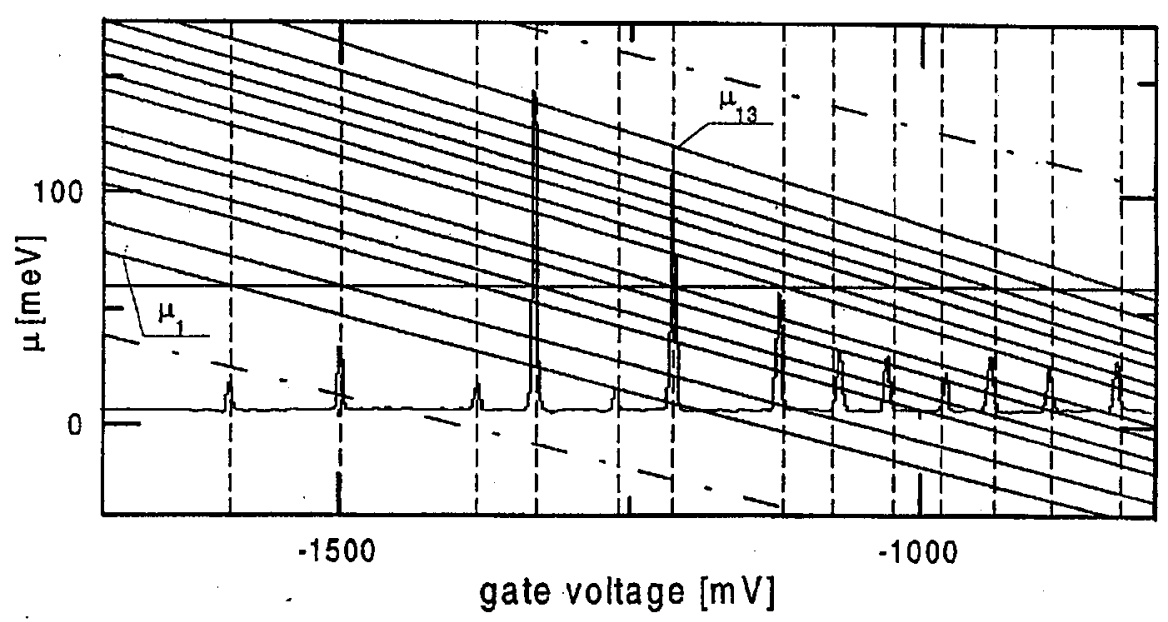

Fig. 1. Chemical potentials $\mu_{N}$ for $N=1, \ldots, 13$ electrons as functions of the potential energy of the gate electrode. The measured [1] current-gate voltage characteristic is also shown. The horizontal solid line shows the Fermi energy of the source-drain system at zero source-drain voltage and the vertical dashed lines show the positions of peaks predicted in the present paper. The lower (upper) dashed line corresponds to the potential-energy minimum inside the QD (barrier) region.

a negligibly small source-drain voltage, the energy levels of the QD are filled by the electrons up to the common Fermi level of the source-drain system. According to the Coulomb blockade model, the $N$-th peak in the current-gate voltage characteristic appears if an alignment takes place between the chemical potential of the $N$-electron artificial atom and the Fermi level of the source-drain system. In this case, the number of electrons confined in the QD oscillates between $N-1$ and $N$. The chemical potential $\mu_{1}$ of one electron, which corresponds to the gate voltage for the first peak, yields the Fermi energy of the source-drain system (cf. horizontal solid line in Fig. 1). We assume that this Fermi energy is independent of the gate voltage. The results of the present calculations exactly reproduce the positions of the first thirteen peaks (Fig. 1) if the parameters of the lateral confinement potential [cf. Eq. (1)] take on the following values: $\alpha=0.129, \beta=2.19 \times 10^{-5} \mathrm{~nm}^{-2}$, and $\gamma=3.75 \times 10^{-8} \mathrm{~nm}^{-4}$. Figure 1 shows the first peaks in the current-gate voltage characteristic observed [1] for the very small source-drain voltage $(250 \mu \mathrm{V})$. Solid curves display the calculated chemical 
potentials $\mu_{1}, \ldots, \mu_{13}$. The two dashed-dotted curves show the dependence of the position of the potential-energy minimum (for $\rho=0$ ) inside the QD region (lower curve) and inside the barrier region (upper: curve) on the applied gate voltage.

In Fig. 1 we see that the separations between the certain peaks increase, namely, between the 2nd and 3rd, 6th and 7th, and 12th and 13th peaks. This effect results from the filling by electrons of the subsequent shells of an artificial atom formed in the QD region. The first three shells are filled by 2,4 , and 6 electrons. These numbers are equal to the degrees of degeneracy of the first three energy levels for the two-dimensional harmonic potential. This fact can be used in the interpretation of the present results, since the qualitative properties of electrons confined in the two-dimensional harmonic potential and the potential corresponding to Eq. (1) are similar. We note that for large numbers of electrons the QD exhibits the classical Coulomb blockade effect [2] with the periodic spacing between the peaks.

We have found that the role of the anharmonic term in Eq. (1) is crucial for a correct quantitative description of the experimental data [1]. Without this term, we can reproduce the positions of the first six peaks in Fig. 1, which corresponds to the filling of the first two shells. The anharmonic potential energy in Eq. (1) leads to a stronger localization of electrons in the central QD region, which increases the chemical potentials of the electronic system. Figure 2 shows the charge density calculated for 12 electrons and the harmonic and anharmonic confinement

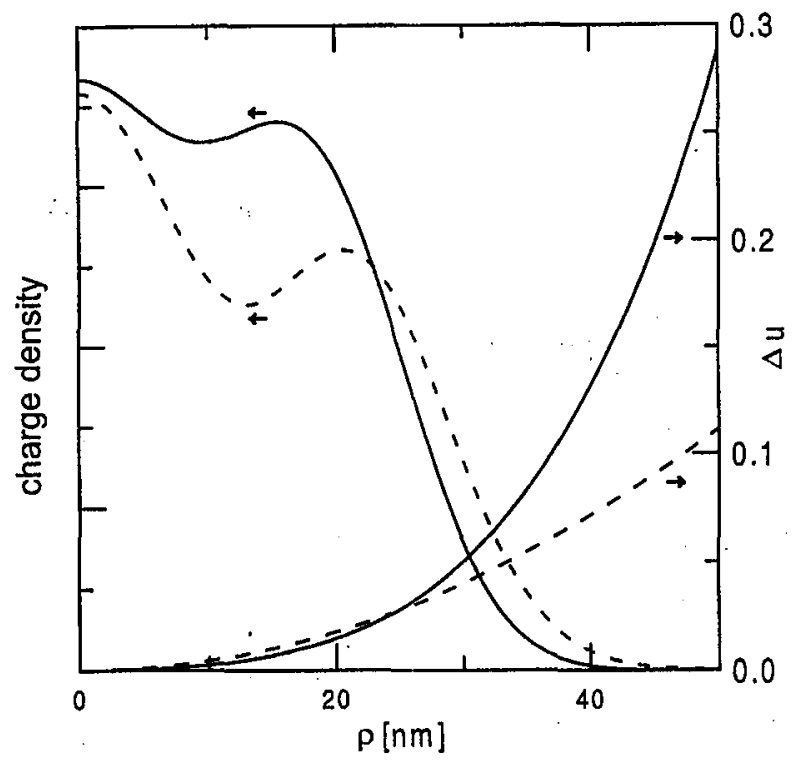

Fig. 2. Charge density (in arbitrary units) calculated for 12 electrons in the QD under the gate voltage $V_{\text {gate }}=-850 \mathrm{mV}$ (left scale) and the values of $\Delta u=$ $\left[U_{\perp}(\rho)-U_{\perp}(0)\right] /\left|e V_{\text {gate }}\right|$ (right scale) as functions of QD radius $\rho$. Solid (dashed) curves correspond to the anharmonic (harmonic) interaction. 
potential. The effect of the stronger electron localization is clearly visible for the anharmonic interaction.

In summary, the present paper provides the first quantitative explanation of the quantum Coulomb blockade in cylindrical quantum dots. This effect results from the quantum many-particle properties of the confined electron system. In a future research, it would be interesting to calculate the confining potential by solving numerically the Poisson equation and to extend the theoretical description to the case of non-zero source-drain voltages.

\section{References}

[1] S. Tarucha, D.G. Austing, T. Honda, R.J. van der Hage, L.P. Kouwenhoven, Phys. Rev. Lett. 77, 3613 (1996).

[2] M. Kastner, Phys. Today 46, 24 (1993).

[3] M. Ezaki, N. Mori, C. Hamaguchi, Phys. Rev. B 56, 6428 (1997).

[4] S. Nagaraya, P. Matagne, V.-Y. Thean, J.-P. Leburton, Y.-H. Kim, R.M. Martin, Phys. Rev. B 56, 15752 (1997).

[5] M. Fujito, A. Natori, H. Yasunaga, Phys. Rev. B 53, 9952 (1996). 